\title{
Evaluating and optimizing the parameters of an unsupervised graph-based WSD algorithm
}

\author{
Eneko Agirre, David Martínez, Oier López de Lacalle and Aitor Soroa \\ IXA NLP Group \\ University of Basque Country \\ Donostia, Basque Contry \\ a.soroadehu.es
}

\begin{abstract}
Véronis (2004) has recently proposed an innovative unsupervised algorithm for word sense disambiguation based on small-world graphs called HyperLex. This paper explores two sides of the algorithm. First, we extend Véronis' work by optimizing the free parameters (on a set of words which is different to the target set). Second, given that the empirical comparison among unsupervised systems (and with respect to supervised systems) is seldom made, we used hand-tagged corpora to map the induced senses to a standard lexicon (WordNet) and a publicly available gold standard (Senseval 3 English Lexical Sample). Our results for nouns show that thanks to the optimization of parameters and the mapping method, HyperLex obtains results close to supervised systems using the same kind of bag-ofwords features. Given the information loss inherent in any mapping step and the fact that the parameters were tuned for another set of words, these are very interesting results.
\end{abstract}

\section{Introduction}

Word sense disambiguation (WSD) is a key enabling technology. Supervised WSD techniques are the best performing in public evaluations, but need large amounts of hand-tagging data. Existing handannotated corpora like SemCor (Miller et al., 1993), which is annotated with WordNet senses (Fellbaum,
1998) allow for a small improvement over the simple most frequent sense heuristic, as attested in the allwords track of the last Senseval competition (Snyder and Palmer, 2004). In theory, larger amounts of training data (SemCor has approx. 500M words) would improve the performance of supervised WSD, but no current project exists to provide such an expensive resource.

Supervised WSD is based on the "fixed-list of senses" paradigm, where the senses for a target word are a closed list coming from a dictionary or lexicon. Lexicographers and semanticists have long warned about the problems of such an approach, where senses are listed separately as discrete entities, and have argued in favor of more complex representations, where, for instance, senses are dense regions in a continuum (Cruse, 2000).

Unsupervised WSD has followed this line of thinking, and tries to induce word senses directly from the corpus. Typical unsupervised WSD systems involve clustering techniques, which group together similar examples. Given a set of induced clusters (which represent word uses or senses ${ }^{1}$ ), each new occurrence of the target word will be compared to the clusters and the most similar cluster will be selected as its sense.

Most of the unsupervised WSD work has been based on the vector space model (Schütze, 1998; Pantel and Lin, 2002; Purandare and Pedersen, 2004), where each example is represented by a vector of features (e.g. the words occurring in the context). Recently, Véronis (Véronis, 2004) has

\footnotetext{
${ }^{1}$ Unsupervised WSD approaches prefer the term 'word uses' to 'word senses'. In this paper we use them interchangeably to refer to both the induced clusters, and to the word senses from some reference lexicon.
} 
proposed HyperLex, an application of graph models to WSD based on the small-world properties of cooccurrence graphs. Hand inspection of the clusters (called hubs in this setting) by the author was very positive, with hubs capturing the main senses of the words. Besides, hand inspection of the disambiguated occurrences yielded precisions over 95\% (compared to a most frequent baseline of 73\%) which is an outstanding figure for WSD systems.

We noticed that HyperLex had some free parameters and had not been evaluated against a public gold standard. Besides, we were struck by the few works where supervised and unsupervised systems were evaluated on the same test data. In this paper we use an automatic method to map the induced senses to WordNet using hand-tagged corpora, enabling the automatic evaluation against available gold standards (Senseval 3 English Lexical Sample S3LS (Mihalcea et al., 2004)) and the automatic optimization of the free parameters of the method. The use of hand-tagged corpora for tagging makes this algorithm a mixture of unsupervised and supervised: the method to induce senses in completely unsupervised, but the mapping is supervised (albeit very straightforward).

This paper is structured as follows. We first present the graph-based algorithm as proposed by Véronis, reviewing briefly the features of smallworld graphs. Section 3 presents our framework for mapping and evaluating the induced hubs. Section 4 introduces parameter optimization. Section 5 shows the experiment setting and results. Section 6 analyzes the results and presents related work. Finally, we draw the conclusions and advance future work.

\section{HyperLex}

Before presenting the HyperLex algorithm itself, we briefly introduce small-world graphs.

\subsection{Small world graphs}

The small-world nature of a graph can be explained in terms of its clustering coefficient and characteristic path length. The clustering coefficient of a graph shows the extent to which nodes tend to form connected groups that have many edges connecting each other in the group, and few edges leading out of the group. On the other side, the characteristic path length represents "closeness" in a graph. See (Watts and Strogatz, 1998) for further details on these characteristics.

Randomly built graphs exhibit low clustering coefficients and are believed to represent something very close to the minimal possible average path length, at least in expectation. Perfectly ordered graphs, on the other side, show high clustering coefficients but also high average path length. According to Watts and Strogatz (1998), small-world graphs lie between these two extremes: they exhibit high clustering coefficients, but short average path lengths.

Barabasi and Albert (1999) use the term "scalefree" to graphs whose degree probability follow a power-law ${ }^{2}$. Specifically, scale free graphs follow the property that the probability $P(k)$ that a vertex in the graph interacts with $k$ other vertices decays as a power-law, following $P(k) \sim k^{-\alpha}$. It turns out that in this kind of graphs there exist nodes centrally located and highly connected, called hubs.

\subsection{The HyperLex algorithm for WSD}

The HyperLex algorithm builds a cooccurrence graph for all pairs of words cooccurring in the context of the target word. Véronis shows that this kind of graph fulfills the properties of small world graphs, and thus possess highly connected components in the graph. The centers or prototypes of these components, called hubs, eventually identify the main word uses (senses) of the target word.

We will briefly introduce the algorithm here, check (Véronis, 2004) for further details. For each word to be disambiguated, a text corpus is collected, consisting of the paragraphs where the word occurs. From this corpus, a cooccurrence graph for the target word is built. Nodes in the graph correspond to the words ${ }^{3}$ in the text (except the target word itself). Two words appearing in the same paragraph are said to cooccur, and are connected with edges. Each edge is assigned with a weight which measures the relative frequency of the two words cooccurring. Specifically, let $w_{i j}$ be the weight of the edge ${ }^{4}$ connecting

\footnotetext{
${ }^{2}$ Although scale-free graphs are not necessarily small worlds, a lot of real world networks are both scale-free and small worlds.

${ }^{3}$ Following Véronis, we only work on nouns for the time being.

${ }^{4}$ Note that the cooccurrence graph is undirected, i.e. $w_{i j}=$
} $w_{j i}$ 
nodes $i$ and $j$, then

$$
\begin{gathered}
w_{i j}=1-\max [P(i \mid j), P(j \mid i)] \\
P(i \mid j)=\frac{\text { freq }_{i j}}{\text { freq }_{j}} \text { and } P(j \mid i)=\frac{\text { freq }_{i j}}{\text { freq }_{i}}
\end{gathered}
$$

The weight of an edge measures how tightly connected the two words are. Words which always occur together receive a weight of 0 . Words rarely cooccurring receive weights close to 1 .

Once the cooccurrence graph is built, a simple iterative algorithm is executed to obtain its hubs. At each step, the algorithm finds the vertex with highest relative frequency ${ }^{5}$ in the graph, and, if it meets some criteria, it is selected as a hub. These criteria are determined by a set of heuristic parameters, that will be explained later in Section 4. After a vertex is selected to be a hub, its neighbors are no longer eligible as hub candidates. At any time, if the next vertex candidate has a relative frequency below a certain threshold, the algorithm stops.

Once the hubs are selected, each of them is linked to the target word with edges weighting 0 , and the Minimum Spanning Tree (MST) of the whole graph is calculated and stored.

The MST is then used to perform word sense disambiguation, in the following way. For every instance of the target word, the words surrounding it are examined and confronted with the MST. By construction of the MST, words in it are placed under exactly one hub. Each word in the context receives a set of scores $s$, with one score per hub, where all scores are 0 except the one corresponding to the hub where it is placed. If the scores are organized in a score vector, all values are 0 , except, say, the $i$-th component, which receives a score $d\left(h_{i}, v\right)$, which is the distance between the hub $h_{i}$ and the node representing the word $v$. Thus, $d\left(h_{i}, v\right)$ assigns a score of 1 to hubs and the score decreases as the nodes move away from the hub in the tree.

For a given occurrence of the target word, the score vectors of all the words in the context are added, and the hub that receives the maximum score is chosen.

\footnotetext{
${ }^{5}$ In cooccurrence graphs, the relative frequency of a vertex and its degree are linearly related, and it is therefore possible to avoid the costly computation of the degree.
}

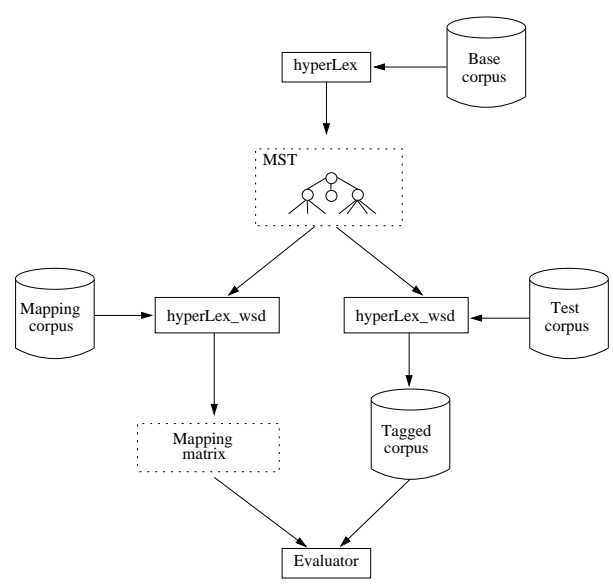

Figure 1: Design for the automatic mapping and evaluation of HyperLex algorithm against a gold standard (test corpora).

\section{Evaluating unsupervised WSD systems}

All unsupervised WSD algorithms need some addition in order to be evaluated. One alternative, as in (Véronis, 2004), is to manually decide the correctness of the hubs assigned to each occurrence of the words. This approach has two main disadvantages. First, it is expensive to manually verify each occurrence of the word, and different runs of the algorithm need to be evaluated in turn. Second, it is not an easy task to manually decide if an occurrence of a word effectively corresponds with the use of the word the assigned hub refers to, especially considering that the person is given a short list of words linked to the hub. We also think that instead of judging whether the hub returned by the algorithm is correct, the person should have independently tagged the occurrence with hubs, which should have been then compared to the hub returned by the system.

A second alternative is to evaluate the system according to some performance in an application, e.g. information retrieval (Schütze, 1998). This is a very attractive idea, but requires expensive system development and it is sometimes difficult to separate the reasons for the good (or bad) performance.

A third alternative would be to devise a method to map the hubs (clusters) returned by the system to the senses in a lexicon. Pantel and Lin (2002) automatically map the senses to WordNet, and then measure the quality of the mapping. More recently, the mapping has been used to test the system on publicly available benchmarks (Purandare and Ped- 


\begin{tabular}{|c|c|cc|cc|cc|}
\hline & $\begin{array}{c}\text { Default } \\
\text { value }\end{array}$ & $\begin{array}{c}\text { p180 } \\
\text { Range }\end{array}$ & Best & \multicolumn{2}{c|}{$\mathrm{p} 1800$} & \multicolumn{2}{c|}{ R6700 } \\
Range & Best & Range & Best \\
\hline \hline p1 & 5 & $2-3$ & 2 & $1-3$ & 2 & $1-3$ & 1 \\
p2 & 10 & $3-4$ & 3 & $2-4$ & 3 & $2-4$ & 3 \\
p3 & 0.9 & $0.7-0.9$ & 0.7 & $0.5-0.7$ & 0.5 & $0.3-0.7$ & 0.4 \\
p4 & 4 & 4 & 4 & 4 & 4 & 4 & 4 \\
p5 & 6 & $6-7$ & 6 & $3-7$ & 3 & $1-7$ & 1 \\
p6 & 0.8 & $0.5-0.8$ & 0.6 & $0.4-0.8$ & 0.7 & $0.6-0.95$ & 0.95 \\
p7 & 0.001 & $0.0005-0.001$ & 0.0009 & $0.0005-0.001$ & 0.0009 & $0.0009-0.003$ & 0.001 \\
\hline
\end{tabular}

Table 1: Parameters of the HyperLex algorithm

ersen, 2004; Niu et al., 2005). See Section 6 for more details on these systems.

Yet another possibility is to evaluate the induced senses against a gold standard as a clustering task. Induced senses are clusters, gold standard senses are classes, and measures from the clustering literature like entropy or purity can be used. As we wanted to focus on the comparison against a standard data-set, we decided to leave aside this otherwise interesting option.

In this section we present a framework for automatically evaluating unsupervised WSD systems against publicly available hand-tagged corpora. The framework uses three data sets, called Base corpus, Mapping corpus and Test corpus:

- The Base Corpus: a collection of examples of the target word. The corpus is not annotated.

- The Mapping Corpus: a collection of examples of the target word, where each corpus has been manually annotated with its sense.

- The Test Corpus: a separate collection, also annotated with senses.

The evaluation framework is depicted in Figure 1. The first step is to execute the HyperLex algorithm over the Base corpus in order to obtain the hubs of a target word, and the generated MST is stored. As stated before, the Base Corpus is not tagged, so the building of the MST is completely unsupervised.

In a second step (left part in Figure 1), we assign a hub score vector to each of the occurrences of target word in the Mapping corpus, using the MST calculated in the previous step (following the WSD algorithm in Section 2.2). Using the hand-annotated sense information, we can compute a mapping matrix $M$ that relates hubs and senses in the following way. Suppose there are $m$ hubs and $n$ senses for the target word. Then, $M=\left\{m_{i j}\right\} \quad 1 \leq i \leq m, 1 \leq$ $j \leq n$, and each $m_{i j}=P\left(s_{j} \mid h_{i}\right)$, that is, $m_{i j}$ is the probability of a word having sense $j$ given that it has been assigned hub $i$. This probability can be computed counting the times an occurrence with sense $s_{j}$ has been assigned hub $h_{i}$.

This mapping matrix will be used to transform any hub score vector $\bar{h}=\left(h_{1}, \ldots, h_{m}\right)$ returned by the WSD algorithm into a sense score vector $\bar{s}=\left(s_{1}, \ldots, s_{n}\right)$. It suffices to multiply the score vector by $M$, i.e., $\bar{s}=\bar{h} M$.

In the last step (right part in Figure 1), we apply the WSD algorithm over the Test corpus, using again the MST generated in the first step, and returning a hub score vector for each occurrence of the target word in the test corpus. We then run the Evaluator, which uses the $M$ mapping matrix in order to convert the hub score vector into a sense score vector. The Evaluator then compares the sense with highest weight in the sense score vector to the sense that was manually assigned, and outputs the precision figures.

Preliminary experiments showed that, similar to other unsupervised systems, HyperLex performs better if it sees the test examples when building the graph. We therefore decided to include a copy of the training and test corpora in the base corpus (discarding all hand-tagged sense information, of course). Given the high efficiency of the algorithm this poses no practical problem (see efficiency figures in Section 6).

\section{Tuning the parameters}

As stated before, the behavior of the HyperLex algorithm is influenced by a set of heuristic parameters, that affect the way the cooccurrence graph is built, the number of induced hubs, and the way they are extracted from the graph. There are 7 parameters in total:

p1 Minimum frequency of edges (occurrences)

p2 Minimum frequency of vertices (words)

p3 Edges with weights above this value are removed

p4 Context containing fewer words are not processed 


\begin{tabular}{|c|c|c|c|c|c|c|c|}
\hline word & train & test & MFS & default & p180 & p1800 & p6700 \\
\hline argument & 221 & 111 & $\overline{51.4}$ & $\overline{51.4}$ & $\overline{51.4}$ & $\overline{51.4}$ & $\overline{51.4}$ \\
\hline arm & 266 & 133 & 82.0 & 82.0 & 80.5 & 82.0 & 82.7 \\
\hline atmosphere & 161 & 81 & 66.7 & 67.9 & 70.4 & 70.4 & 67.9 \\
\hline audience & 200 & 100 & 67.0 & 69.0 & 71.0 & 74.0 & 77.0 \\
\hline bank & 262 & 132 & 67.4 & 69.7 & 75.0 & 76.5 & 75.0 \\
\hline degree & 256 & 128 & 60.9 & 60.9 & 60.9 & 62.5 & 63.3 \\
\hline difference & 226 & 114 & 40.4 & 40.4 & 41.2 & 46.5 & 49.1 \\
\hline difficulty & 46 & 23 & 17.4 & 30.4 & 30.4 & 39.1 & 26.1 \\
\hline disc & 200 & 100 & 38.0 & 66.0 & 75.0 & 70.0 & 76.0 \\
\hline image & 146 & 74 & 36.5 & 63.5 & 62.2 & 67.6 & 64.9 \\
\hline interest & 185 & 93 & 41.9 & 49.5 & 41.9 & 47.3 & 51.6 \\
\hline judgment & 62 & 32 & 28.1 & 28.1 & 28.1 & 53.1 & 50.0 \\
\hline organization & 112 & 56 & 73.2 & 73.2 & 73.2 & 71.4 & 73.2 \\
\hline paper & 232 & 117 & 25.6 & 42.7 & 39.3 & 47.9 & 53.8 \\
\hline party & 230 & 116 & 62.1 & 67.2 & 64.7 & 65.5 & 67.2 \\
\hline performance & 172 & 87 & 32.2 & 44.8 & 46.0 & 54.0 & 59.8 \\
\hline plan & 166 & 84 & 82.1 & 81.0 & 79.8 & 81.0 & 83.3 \\
\hline shelter & 196 & 98 & 44.9 & 45.9 & 49.0 & 48.0 & 54.1 \\
\hline sort & 190 & 96 & 65.6 & 64.6 & 64.6 & 65.6 & 64.6 \\
\hline source & 64 & 32 & 65.6 & 59.4 & 56.2 & 62.5 & 62.5 \\
\hline & & Average: & 254.5 & $\overline{59.9}$ & 60.3 & 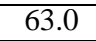 & 64.6 \\
\hline & & ver S2LS) & 51.9 & 56.2 & 57.5 & 58.7 & 60.0 \\
\hline
\end{tabular}

Table 2: Precision figures for nouns over the test corpus (S3LS). The second and third columns show the number of occurrences in the train and test splits. The MFS column corresponds to the most frequent sense. The rest of columns correspond to different parameter settings: default for the default setting, $p 180$ for the best combination over 180, etc.. The last rows show the microaverage over the S3LS run, and we also add the results on the S2LS dataset (different sets of nouns) to confirm that the same trends hold in both datasets.

p5 Minimum number of adjacent vertices a hub must have p6 Max. mean weight of the adjacent vertices of a hub p7 Minimum frequency of hubs

Table 1 lists the parameters of the HyperLex algorithm, and the default values proposed for them in the original work (second column).

Given that we have devised a method to efficiently evaluate the performance of HyperLex, we are able to tune the parameters against the gold standard. We first set a range for each of the parameters, and evaluated the algorithm for each combination of the parameters on a collection of examples of different words (Senseval 2 English lexical-sample, S2LS). This ensures that the chosen parameter set is valid for any noun, and is not overfitted to a small set of nouns. ${ }^{6}$ The set of parameters that obtained the best results in the S2LS run is then selected to be run against the S3LS dataset.

We first devised ranges for parameters amounting to 180 possible combinations (p180 column in Table 2), and then extended the ranges to amount to 1800 and 6700 combinations (columns p1800 and p6700).

\footnotetext{
${ }^{6}$ In fact, previous experiments showed that optimizing the parameters for each word did not yield better results.
}

\section{Experiment setting and results}

To evaluate the HyperLex algorithm in a standard benchmark, we applied it to the 20 nouns in S3LS. We use the standard training-test split. Following the design in Section 3, we used both the training and test sets as the Base Corpus (ignoring the sense tags, of course). The Mapping Corpus comprised the training split only, and the Test corpus the test split only. The parameter tuning was done in a similar fashion, but on the S2LS dataset.

In Table 2 we can see the number of examples of each word in the different corpus and the results of the algorithm. We indicate only precision, as the coverage is $100 \%$ in all cases. The left column, named MFS, shows the precision when always assigning the most frequent sense (relative to the train split). This is the baseline of our algorithm as our algorithm does see the tags in the mapping step (see Section 6 for further comments on this issue).

The default column shows the results for the HyperLex algorithm with the default parameters as set by Véronis, except for the minimum frequency of the vertices ( $p 2$ in Table 1), which according to some preliminary experiments we set to 3 . As we can see, the algorithm with the default settings outperforms 


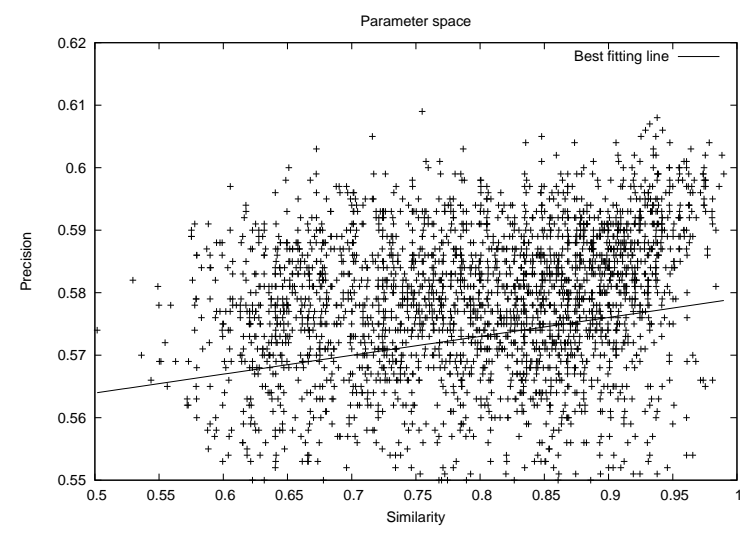

Figure 2: Dispersion plot of the parameter space for 6700 combinations. The horizontal axis shows the similarity of a parameter set w.r.t. the best parameter set using the cosine. The vertical axis shows the precision in S2LS. The best fitting line is also depicted.

the MFS baseline by 5.4 points average, and in almost all words (except plan, sort and source).

The results for the best of 180 combinations of the parameters improve the default setting ( 0.4 overall), Extending the parameter space to 1800 and $6700 \mathrm{im}$ proves the precision up to 63.0 and $64.6,10.1$ over the MFS (MFS only outperforms HyperLex in the best setting for two words). The same trend can be seen on the S2LS dataset, where the gain was more modest (note that the parameters were optimized for S2LS).

\section{Discussion and related work}

We first comment the results, doing some analysis, and then compare our results to those of Véronis. Finally we overview some relevant work and review the results of unsupervised systems on the S3LS benchmark.

\subsection{Comments on the results}

The results show clearly that our exploration of the parameter space was successful, with the widest parameter space showing the best results.

In order to analyze whether the search in the parameter space was making any sense, we drew a dispersion plot (see Figure 2). In the top right-hand corner we have the point corresponding to the best performing parameter set. If the parameters were not conditioning the good results, then we would have expected a random cloud of points. On the contrary, we can see that there is a clear tendency for those

\begin{tabular}{|c|c|c|c|c|}
\hline & default & p180 & p1800 & p6700 \\
\hline hubs defined & $\overline{9.2 \pm 3.8}$ & $\overline{15.3 \pm 5.7}$ & $\overline{\overline{38.6 \pm 11.8}}$ & $\overline{777.7 \pm 18.7}$ \\
\hline used & $8.4 \pm 3.5$ & $14.4 \pm 5.3$ & $30.4 \pm 9.3$ & $45.2 \pm 13.3$ \\
\hline senses defined & $5.4 \pm 1.5$ & $5.4 \pm 1.5$ & $5.4 \pm 1.5$ & $5.4 \pm 1.5$ \\
\hline used & $2.6 \pm 1.2$ & $2.5 \pm 1$ & $3.1 \pm 1.1$ & $3.2 \pm 1.2$ \\
\hline senses in tes & $5.1 \pm 1.3$ & - & - & - \\
\hline
\end{tabular}

Table 3: Average number of hubs and senses (along with the standard deviation) for three parameter settings. Defined means the number of hubs induced, and used means the ones actually returned by HyperLex when disambiguating the test set. The same applies for senses, that is, defined means total number of senses (equal for all columns), and used means the senses that were actually used by HyperLex in the test set. The last row shows the actual number of senses used by the hand-annotators in the test set.

parameter sets most similar to the best one to obtain better results, and in fact the best fitting line shows a clearly ascending slope.

Regarding efficiency, our implementation of HyperLex is extremely fast. Doing the 1800 combinations takes 2 hours in a 2 AMD Opteron processors at $2 \mathrm{GHz}$ and $3 \mathrm{~Gb}$ RAM. A single run (building the MST, mapping and tagging the test sentences) takes only $16 \mathrm{sec}$. For this reason, even if an on-line version would be in principle desirable, we think that this batch version is readily usable.

\subsection{Comparison to (Véronis, 2004)}

Compared to Véronis we are inducing larger numbers of hubs (with different parameters), using less examples to build the graphs and obtaining more modest results (far from the 90's). Regarding the latter, our results are in the range of other S3LS WSD systems (see below), and the discrepancy can be explained by the way Véronis performed his evaluation (see Section 3).

Table 3 shows the average number of hubs for the four parameter settings. The average number of hubs for the default setting is larger than that of Véronis (which ranges between 4 and 9 per word), but quite close to the average number of senses. The exploration of the parameter space prefers parameter settings with even larger number of hubs, and the figures shows that most of them are actually used for disambiguation. The table also shows that, after the mapping, less than half of the senses are actually used, which seems to indicate that the mapping tends to favor the most frequent senses.

Regarding the actual values of the parameters used (c.f. Table 1), we had to reduce the value 
of some parameters (e.g. the minimum frequency of vertices) due to the smaller number of of examples (Véronis used from 1900 to 8700 examples per word). In theory, we could explore larger parameter spaces, but Table 1 shoes that the best setting for the 6700 combinations has no parameter in a range boundary (except $p 5$, which cannot be further reduced).

All in all, the best results are attained with smaller and more numerous hubs, a kind of micro-senses. A possible explanation for this discrepancy with Véronis could be that he was inspecting by hand the hubs that he got, and perhaps was biased by the fact that he wanted the hubs to look more like standard senses. At first we were uncomfortable with this behavior, so we checked whether HyperLex was degenerating into a trivial solution. We simulated a clustering algorithm returning one hub per example, and its precision was 40.1, well below the MFS baseline. We also realized that our results are in accordance with some theories of word meaning, e.g. the "indefinitely large set of prototypes-withinprototypes" envisioned in (Cruse, 2000). We now think that the idea of having many micro-senses is very attractive for further exploration, especially if we are able to organize them into coarser hubs.

\subsection{Comparison to related work}

Table 4 shows the performance of different systems on the nouns of the S3LS benchmark. When not reported separately, we obtained the results for nouns running the official scorer program on the filtered results, as available in the S3LS web page. The second column shows the type of system (supervised, unsupervised).

We include three supervised systems, the winner of S3LS (Mihalcea et al., 2004), an in-house system (kNN-all, CITATION OMITTED) which uses optimized $\mathrm{kNN}$, and the same in-house system restricted to bag-of-words features only (kNN-bow), i.e. discarding other local features like bigrams or trigrams (which is what most unsupervised systems do). The table shows that we are one point from the bag-ofwords classifier kNN-bow, which is an impressive result if we take into account the information loss of the mapping step and that we tuned our parameters on a different set of words. The full $\mathrm{kNN}$ system is state-of-the-art, only 4 points below the S3LS win-

\begin{tabular}{|l|c|c|c|}
\hline System & Type & Prec. & Cov. \\
\hline S3LS-best & Sup. & 74.9 & 0.99 \\
kNN-all & Sup. & 70.3 & 1.0 \\
kNN-bow & Sup. & 65.7 & 1.0 \\
HyperLex & Unsup(S3LS) & 64.6 & 1.0 \\
Cymfony & Unsup(10\%-S3LS) & 57.9 & 1.0 \\
Prob0 & Unsup. (MFS-S3) & 55.0 & 0.98 \\
MFS & - & 51.5 & 1.0 \\
Ciaosenso & Unsup (MFS-Sc) & 53.95 & 0.90 \\
clr04 & Unsup (MFS-Sc) & 48.86 & 1.0 \\
duluth-senserelate & Unsup & 47.48 & 1.0 \\
\hline (Purandare and & Unsup (S2LS) & - & - \\
Pedersen, 2004) & & & \\
\hline
\end{tabular}

Table 4: Comparison of HyperLex and MFS baseline to S3LS systems for nouns. The last system was evaluated on S2LS.

ner.

Table 4 also shows several unsupervised systems, all of which except Cymfony and (Purandare and Pedersen, 2004) participated in S3LS (check (Mihalcea et al., 2004) for further details on the systems). We classify them according to the amount of "supervision" they have: some have have access to most-frequent information (MFS-S3 if counted over S3LS, MFS-Sc if counted over SemCor), some use $10 \%$ of the S3LS training part for mapping (10\%S3LS), and some use the full amount of S3LS training for mapping (S3LS). Only one system (Duluth) did not use in any way hand-tagged corpora.

Given the different typology of unsupervised systems, it's unfair to draw definitive conclusions from a raw comparison of results. The system coming closer to ours is that described in (Niu et al., 2005). They use hand tagged corpora which does not need to include the target word to tune the parameters of a rather complex clustering method which does use local information (an exception to the rule of unsupervised systems). They do use the S3LS training corpus for mapping. For every sense the target word, three of its contexts in the train corpus are gathered (around 10\% of the training data) and tagged. Each cluster is then related with its most frequent sense. Only one cluster may be related to a specific sense, so if two or more clusters map to the same sense, only the largest of them is retained. The mapping method is similar to ours, but we use all the available training data and allow for different hubs to be assigned to the same sense.

Another system similar to ours is (Purandare and Pedersen, 2004), which unfortunately was evaluated on Senseval 2 data. The authors use first and second 
order bag-of-word context features to represent each instance of the corpus. They apply several clustering algorithms based on the vector space model, limiting the number of clusters to 7 . They also use all available training data for mapping, but given their small number of clusters they opt for a one-to-one mapping which maximizes the assignment and discards the less frequent clusters. They also discard some difficult cases, like senses and words with low frequencies $(10 \%$ of total occurrences and 90, respectively). The different test set and mapping system make the comparison difficult, but the fact that the best of their combinations beats MFS by 1 point on average $(47.6 \%$ vs. $46.4 \%)$ for the selected nouns and senses make us think that our results are more robust (nearly $10 \%$ over MFS).

\section{Conclusions and further work}

This paper has explored two sides of HyperLex: the optimization of the free parameters, and the empirical comparison on a standard benchmark against other WSD systems. We use hand-tagged corpora to map the induced senses to WordNet senses.

Regarding the optimization of parameters, we used a another testbed (S2LS) comprising different words to select the best parameter. We consistently improve the results of the parameters by Véronis, which is not perhaps so surprising, but the method allows to fine-tune the parameters automatically to a given corpus given a small test set.

Comparing unsupervised systems against supervised systems is seldom done. Our results indicate that HyperLex with the supervised mapping is on par with a state-of-the-art system which uses bagof-words features only. Given the information loss inherent to any mapping, this is an impressive result. The comparison to other unsupervised systems is difficult, as each one uses a different mapping strategy and a different amount of supervision.

For the future, we would like to look more closely the micro-senses induced by HyperLex, and see if we can group them into coarser clusters. We also plan to apply the parameters to the Senseval 3 allwords task, which seems well fit for HyperLex: the best supervised system only outperforms MFS by a few points in this setting, and the training corpora used (Semcor) is not related to the test corpora (mainly Wall Street Journal texts).

Graph models have been very successful in some settings (e.g. the PageRank algorithm of Google), and have been rediscovered recently for natural language tasks like knowledge-based WSD, textual entailment, summarization and dependency parsing. We would like to test other such algorithms in the same conditions, and explore their potential to integrate different kinds of information, especially the local or syntactic features so successfully used by supervised systems, but also more heterogeneous information from knowledge bases.

\section{References}

A. L. Barabasi and R. Albert. 1999. Emergence of scaling in random networks. Science, 286(5439):509-512, October.

D. A. Cruse, 2000. Polysemy: Theoretical and Computational Approaches, chapter Aspects of the Microstructure of Word Meanings. OUP.

C. Fellbaum. 1998. WordNet: An Electronic Lexical Database. MIT Press.

R. Mihalcea, T. Chklovski, and A. Kilgarriff. 2004. The senseval-3 english lexical sample task. In R. Mihalcea and P. Edmonds, editors, Senseval-3 proceedings, pages 25-28. ACL, July.

G.A. Miller, C. Leacock, R. Tengi, and R.Bunker. 1993. A semantic concordance. In Proc. of the ARPA HLT workshop.

C. Niu, W. Li, R. K. Srihari, and H. Li. 2005. Word independent context pair classification model for word sense disambiguation. In Proc. of CoNLL-2005.

P. Pantel and D. Lin. 2002. Discovering word senses from text. In Proc. of KDD02.

A. Purandare and T. Pedersen. 2004. Word sense discrimination by clustering contexts in vector and similarity spaces. In Proc. of CoNLL-2004.

H. Schütze. 1998. Automatic word sense discrimination. Computational Linguistics, 24(1):97-123.

B. Snyder and M. Palmer. 2004. The english all-words task. In Proc. of SENSEVAL.

J. Véronis. 2004. HyperLex: lexical cartography for information retrieval. Computer Speech \& Language, 18(3):223-252.

D. J. Watts and S. H. Strogatz. 1998. Collective dynamics of 'small-world' networks. Nature, 393(6684):440-442, June. 